Delia Cortés-Guiral*, Olivia Sgarbura, Mohammad Alyami, Kazuhiro Yoshida, Yuichiro Doki, Hironori Ishigami, Fabian Grass and Martin Hübner

\title{
Priorities, actions and risks in the COVID-19 pandemic: a flash SoMe survey among surgical oncologists
}

https://doi.org/10.1515/pp-2020-0142

Received September 4, 2020; accepted November 2, 2020;

published online January 25, 2021

\section{Abstract}

Objectives: Corona virus-induced disease 19 (COVID-19) pandemic has globally affected the surgical treatment of cancer patients and has challenged the ethical principles of surgical oncologists around the world. Not only treatment but also diagnosis and follow-up have been disrupted.

Methods: An online survey was sent through Twitter and by the surgical societies worldwide. The survey consisted of 29 closed-ended questions and was conducted over a period of 24 days beginning in March 26, 2020.

Results: Overall, 394 surgical oncologists from 41 different countries answered the questionnaire. The predominant guiding principle was "saving lives" 240 (62\%), and the different aspects of lock-down found hence large support (mean 7.1-9.3 out of 10). Shut-down of elective surgery and modification of cancer care found a mean support of $7.0 \pm 3.0$ and $5.8 \pm 3.1$, respectively. Modification of cancer

*Corresponding author: Delia Cortés-Guiral, Department of General Surgery and Surgical Oncology, Oncology Center, King Khalid University Hospital, Najran, Saudi Arabia,

E-mail: delia.cortes.guiral@gmail.com. https://orcid.org/00000002-3834-2271

Olivia Sgarbura, Department of Surgical Oncology, Cancer Institute Montpellier (ICM), Montpellier, France; and University of Montpellier, Montpellier, France. https://orcid.org/0000-0002-6965-3697 Mohammad Alyami, Department of General Surgery and Surgical Oncology, King Khalid Hospital, Najran, Saudi Arabia

Kazuhiro Yoshida, Department of Surgical Oncology, Gifu University School of Medicine, Gifu, Japan

Yuichiro Doki, Department of Gastroenterological Surgery, Graduate School of Medicine, Osaka University, Suita, Osaka, Japan

Hironori Ishigami, Department of Surgical Oncology, The University of Tokyo, Tokyo, Japan

Fabian Grass, Division of Colon and Rectal Surgery, Mayo Clinic, Rochester, MN, USA; and Department of Visceral Surgery, Lausanne University Hospital CHUV, University of Lausanne (UNIL), Lausanne, Switzerland

Martin Hübner, Department of Visceral Surgery, Lausanne University Hospital CHUV, University of Lausanne (UNIL), Lausanne, Switzerland care longer than two weeks was considered unacceptable to $114(29 \%)$ responders. Hundred and fifty six $(40 \%)$ and 138 (36\%) expect "return to normal" beyond six months for surgical practice and cancer care, respectively.

Conclusions: Surgical oncologists show strong and longlasting support for lock-down measures aiming to save lives. The impact of the pandemic on surgical oncology is perceived controversially, but the majority was forced already now to accept what is inacceptable for many of their colleagues.

Keywords: COVID-19; priorities; surgical oncology.

\section{Introduction}

Corona virus-induced disease 19 (COVID-19) pandemic changed our professional and private lives [1-3] since a brief report to WHO China of few cases of respiratory pneumonia of unknown origin at the end of December 2019 [4] turned into a declaration of Public Health Emergency of International Concern (PHEIC) on January 30 [5]. Radical measures needed to be taken in most countries to contain infectious spread and to increase or shift resources from most domains to emergency units and intensive care aiming to avoid to increase the surge capacity of health care systems and to minimize the number of avoidable COVID-19 deaths [6-16].

The general principle of patient care "primum not nocere" was challenged in COVID-19 times, as some of these measures required to limit surgical service and to delay or modify cancer care with yet unknown consequences for the concerned patients [17-21].

The aim of this survey was to study the surgical oncologist's view on priorities, risks and measures during the COVID-19 pandemic with special focus on the impact on surgical oncology.

\section{Materials and methods}

The present study is a flash survey among surgical oncologists diffused by Social Media (SoMe). The questionnaire consisted of 27 closed-ended questions on demographics $(n=5)$, (A) guiding principles 
$(n=2),(B)$ measures of containment $(n=10)$, (C) shifting of resources, current management $(n=6)$ and (D) perspective $(n=4)$ (Supplementary Material, Appendix p 1). The intuitive online questionnaire (Google Docs ${ }^{\circledR}$ application, 2012, Mountain View, California, USA) could be answered from any connected device (mobile phone, personal computer, tablet and laptop) and took a median of $7 \mathrm{~min}$ to be completed.

The survey was launched online on March 26, 2020 while the pandemic spread exponentially [22-24] and was closed on April 19, when death tolls peaked in the United States [25-27]. Twitter is the most engaged social media application for physicians around the world $[28,29]$ and was therefore chosen as platform to launch the survey. The survey was diffused through the SoMe4Peritoneum account @SPeritoneum, SoMe4Surgery @me4_so and SoMe4HPB @hpb_so (Twitter official accounts from SoMe4Surgery communities specifically focused on surgical oncology and peritoneal surface malignancies) and the private account of one of the surgical oncologists in charge of this community (@DeliaCortesGuir). Concurrently the survey was endorsed and distributed by the following surgical societies: ACS (American College of Surgeons), ESSO (European Society of Surgical Oncology), JSCO (Japan Society Clinical Oncology), RENAPE (French Registry for Rare Peritoneal Tumors), SEOQ (Sociedad Española de Oncología Quirúrgica) and SSCRS (Saudi Society of Colon and Rectal Surgery).

Plain descriptive statistics were used to present the results of the current survey and the frequencies were reported as raw numbers and percentages. Median and mean values were calculated for discrete and continuous variables, respectively. Data analysis was performed with IBM@ SPSS ${ }^{\circledR}$ Statistics Subscription (IBM Inc., Armonk, NY, USA). Discrete variables were compared with Wilcoxon test, categorical variables were compared with $\chi^{2}$-test and correlations were performed with Pearson and Spearman tests. A two-sided alpha of 0.05 was used to indicate statistical significance.

\section{Results}

In the 24 day time period, 394 surgical oncologists from 41 different countries answered the questionnaire. Complete demographics are provided as Supplementary Material, Appendix p. 6. Briefly, 268 (68\%) responders worked in academic institutions or dedicated cancer centers. Most responders were consultants $297(76 \%)$ and had at least one subspecialty 334 (85\%).

\section{Guiding principles}

Figure 1 displays the wide variation of priorities among surgical oncologists concerning pertinent guiding principles. As a pattern, three different levels of priority aims could be identified: 1 . Saving lives, 2. Saving life-years, protection of health care system and workers, 3. Maintaining normal life and limit economic consequences. Of note, two-thirds acknowledged that these declared priorities were likely to change in the future course of the pandemic.

A. Measures of containment

Restrictive measures found variable support by the responders and were considered inevitable by $298(76 \%)$ panelists for canceling of large public events, 244 (62\%) for limitation of travel, 221 (56\%) for closure of schools, 140 (36\%) for lock-down and 107 (28\%) for handy-tracking. Mean support for these measures of containment is displayed in Figure 2. A variable majority (55-76\%) supported all of these measures for "as long as necessary".

B. Shifting resources

Large heterogeneity was encountered with regards to the shutdown of elective surgery program (mean support $7.0 \pm 3.0$ ) and modification of cancer care (Figure 3A, B). The latter found little support by surgical oncologists (mean support $5.8 \pm 3.1$ ). Interestingly $114(29 \%)$ of responders judged modification of cancer care to be inacceptable at all or for >two weeks, while 115 (29\%) could accept this profound paradigm shift for "as long as necessary”. Further, 258 (66\%) declared to have changed their strategy already as detailed in Figure 4.

\section{Perspectives}

Estimated duration until "return to normal" for surgical practice, cancer care, medical meetings and social life are summarized in Table 1. Of note, return to normal surgical practice and cancer care was not expected to occur before six months of time by $156(40 \%)$ and 138 (36\%) of the surveyed surgical oncologists, respectively.

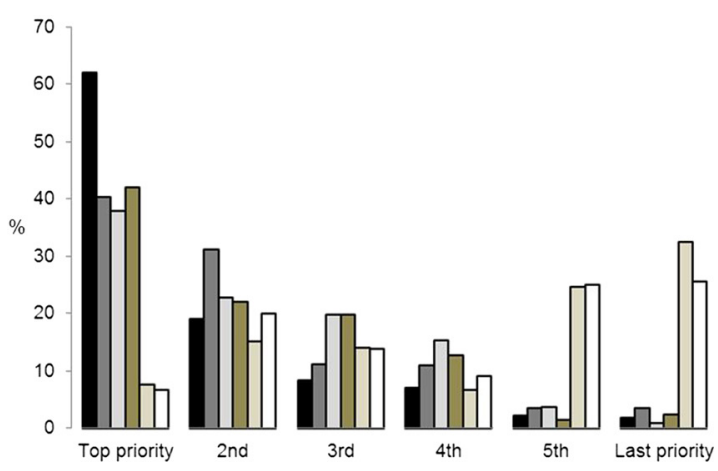

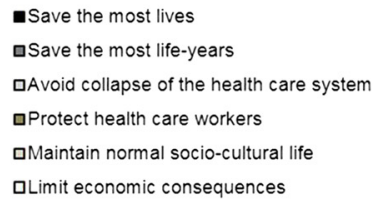

Figure 1: Guiding principles during COVID-19 pandemic. Schematic representation of guiding principles during COVID-19 pandemic ranked by priority. 


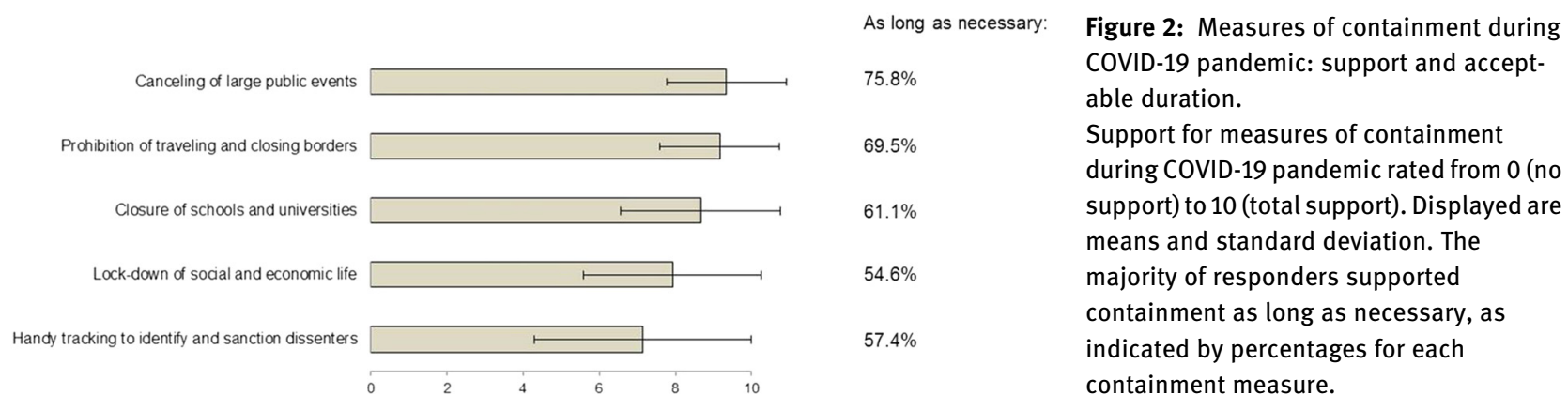

a

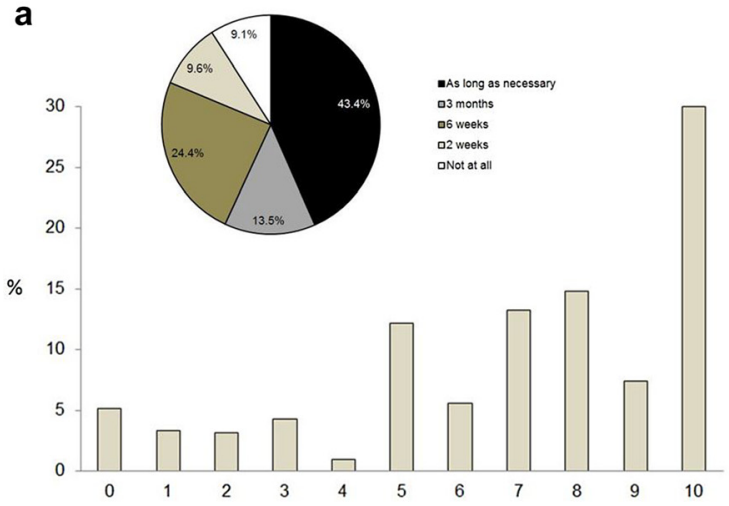

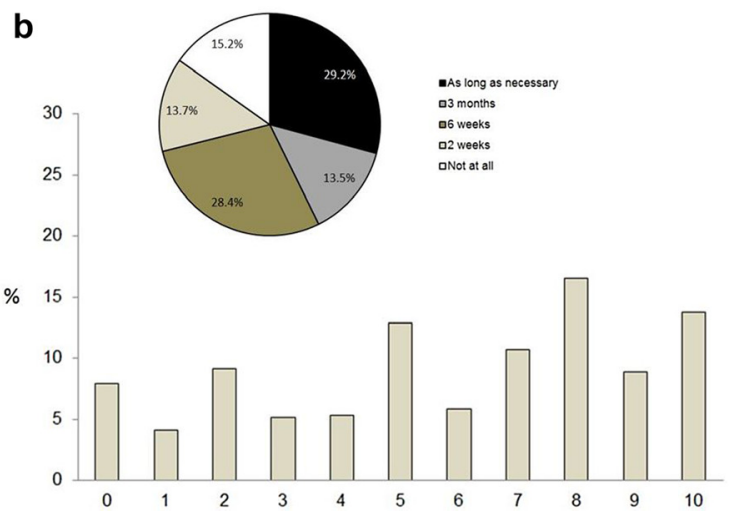

Figure 3: Acceptance of shifting of resources during COVID-19 pandemic.

(A) Shut-down of elective surgery program. (B) Deferring or modification of cancer care. Acceptance of shifting of resources for (A) shut-down of elective surgery program and (B) deferring or modification of cancer care rated from 0 (no support) to 10 (total support). Pie charts represent acceptable time lines for shifting of resources.

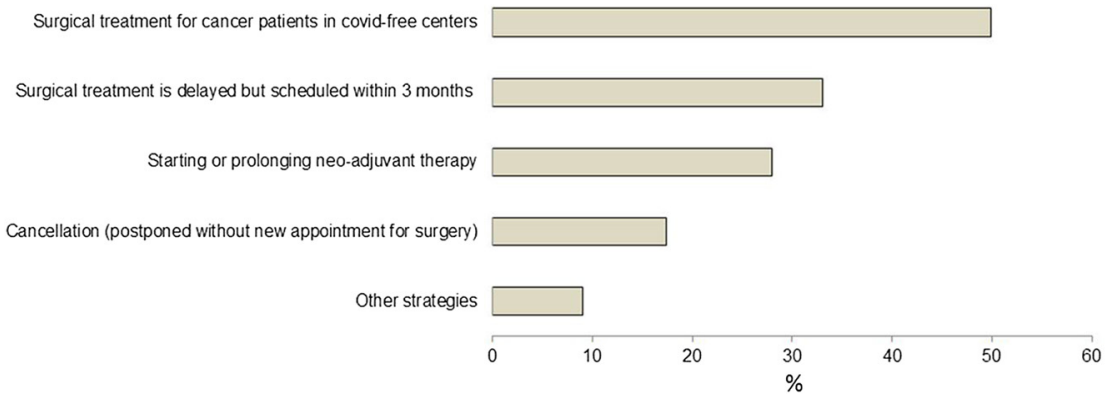

Figure 4: Changes in management of cancer patients during the COVID-19 pandemic.

Table 1: Estimated duration of COVID-19 crisis: "Back to normal".

\begin{tabular}{lrrrr}
\hline Normality in ... & Six weeks & Three months & Six months & One year + \\
\hline Surgical & $24 \%$ & $37 \%$ & $28 \%$ & $12 \%$ \\
practice & & & & \\
Cancer care & $28 \%$ & $37 \%$ & $25 \%$ & $11 \%$ \\
Meetings & $7 \%$ & $21 \%$ & $46 \%$ & $26 \%$ \\
Social life & $8 \%$ & $31 \%$ & $37 \%$ & $24 \%$ \\
\hline
\end{tabular}

Highest proportion is displayed in bold.
D. Intercultural variations

The results of the main group of respondents (Japan) were compared to the rest of the participants. Of note, a significantly lower rate of surgeons needed to change their strategies of cancer care (31 vs. $88 \%, \mathrm{p}<0.0001)$ but a similar number estimated that they might change their priorities in the future (62 vs. $70 \%$ in the rest of the group, $\mathrm{p}=$ not significant [NS]). The ranking of priorities was similar in the two populations. Global support for the 
measures of containment was significantly lower in the Japanese population ( 8 vs. 9.2, p<0.0001) and the difference was confirmed for every category.

Support for deferring cancer care was significantly lower than for all other measures of containment in the entire population ( 5.8 vs 8.7 ; $\mathrm{p}<0.0001$ ). The support was lower in the Japanese subgroup than in the rest of the respondents (5.3 vs 6.3; $\mathrm{p}<0.0001$ ).

\section{Discussion}

The present flash survey depicts the current view of surgical oncologists on priorities, measures and their impact on surgical care. The top priority guiding principle "to safe lives" leads currently to wide acceptance of restrictive measures despite the perceived negative impact on cancer care. While there is reluctance to delay or modify surgical oncology, this change has widely taken place already and it is expected to last for at least six months.

Fair allocation of resources in situation of scarcity has been long discussed in different contexts $[30,31]$ and it is nowadays accepted that the four leading principles are: maximizing benefits of scarce resources, treating people equally, promoting and rewarding instrumental value and giving priority to the worst off. In this survey, "saving most lives" came up as a top priority for most respondents while "saving most life-years" appeared more frequently in the second position but was almost as frequently cited as the third or even fourth priority. These two principles are considered as the equivalent of a consensus in bioethics [30-32] and, although some heterogeneity was noted, surgical oncologists recognized them as such.

Although the support of surgical oncologists for different containment measures was variable, cancelation of public events, limitations of travel and closure of schools obtained a moderate to large consensus (more than $60 \%$ ) just as a similar rate was encountered in support of these measures "as long as necessary". These results are consistent with the fact that surgical oncologists are caregivers placing healthcare in the top of the societal prime concern, particularly in pandemic context. Given the large range of nationalities of the respondents of these surveys and the share of $38 \%$ of Japanese oncologists, these results imply that professional focus outreaches the cultural specificity in this issue. Since the first report indicating a significantly higher rate of COVID-19 infections in cancer patients [20], several national and international guidelines proposed recommendations concerning the potential deferral mechanisms in cancer care and targeted pathologies [33-36]. In the light of these recommendations, some authors $[37,38]$ analyzed big data in a tentative to calculate a safe postponement period that turned out to be a median of three weeks since specialist consultation and six weeks since diagnosis for cancers treated with surgery first. In the present survey, two-thirds of the respondents acknowledge to have changed their cancer care strategies during the COVID-19 pandemic. The acceptable deferring period for cancer care was the item with the highest heterogeneity in this study. Of note, one third of the respondents considered that deferral was unacceptable or acceptable for a maximum of two weeks while another third accepted it for "as long as necessary". These results are highly suggestive of a potential conflict faced by surgical oncologists during the present sanitary situations as the traditional pressure put on cancer care systems to be highly responsive in the management of the disease collides with present ambiguous recommendations from scientific societies and scant evidence-based data.

Even in normal times, rationing of the resources and, potentially, of healthcare is unavoidable [39]. However, it has been showed before that practitioners, including ICU practitioners that are exposed to that selection on a regular basis, are not always aware of the choices they are making [39]. Furthermore, the individual physician should not be faced with the terrible task of performing the selection in isolation [30]. The current situation is sometimes exposing the surgical oncologists to making that choice on their own or together with their colleagues in local multidisciplinary tumor boards which questions their preparation to facing bioethical dilemmas.

In the present flash survey, two-thirds of the respondents admitted that they might change priorities during this sanitary situation and more than a third did not think that a return to normality would happen before six months. These results are in contrast with the reduced acceptability of the cancer care deferral, but they show that, in spite of the lack of explicit knowledge, surgical oncologists remain highly adaptable to change. In Megginson's words “According to Darwin's Origin of Species, it is not the most intellectual of the species that survives; it is not the strongest that survives; but the species that survives is the one that is able best to adapt and adjust to the changing environment in which it finds itself " [40].

The present survey benefitted from a high rate of Japanese respondents. Their availability to complete the survey is probably linked to the high influence of JSCO. Several highquality Western organizations supported this survey but their area of influence is probably more heterogenous. The earlier phase in the management of COVID-19 at the time of the survey and the significantly lower percentage of Japanese 
surgical oncologists that had to alter cancer care explain lower acceptance of containment measures but probably should not be considered as the sole influencing factor as other cultural and social confounding variable were not taken into consideration by the present survey.

This flash survey can only picture the current situation. Attitudes of responders are likely to change during the pandemic as two-thirds of them confirmed. The surveyed sample of surgical oncologists was not specifically selected (biased) but is not representative neither due to the voluntary character. Response for most of the questions depended on many factors such as country of origin, setting, experienced phase of the pandemic, timing of response to the survey, but also general attitude, personality and values of the responders.

\section{Conclusions}

Surgical oncologists have widely embraced the consequences of prioritizing the principle of "maximizing the benefits", and strong and long-lasting support for lockdown measures aiming to save lives as top guiding principle. The pandemic forced surgical oncologist to accept the inacceptable, namely to modify best cancer care, probably for a prolonged period. To solve this dilemma, alternative strategies need not only to be developed but also to be monitored carefully to guarantee equipoise.

Acknowledgments: The authors thank ACS (American College of Surgeons), ESSO (European Society of Surgical Oncology) , JSCO (Japanese Society of Clinical Oncology), RENAPE (French Registry for Rare Peritoneal Tumors), SEOQ (Spanish Society of Surgical Oncology) and SSCRS (Saudi Society of Colon and Rectal Surgery) for their invaluable contribution and support spreading disinterestedly this survey among their affiliates.

Research funding: None declared.

Author contributions: DCG, OS, MA and MH developed the idea. All authors drafted the survey, the article and prepared the tables and figures. All authors substantially revised the manuscript. All authors have accepted responsibility for the entire content of this manuscript and approved its submission.

Competing interests: Authors state no conflict of interest. Informed consent: Informed consent was obtained from all individuals included in this study

Ethical approval: Research involving human subjects complied with all relevant national regulations, institutional policies and is in accordance with the tenets of the Helsinki Declaration (as revised in 2013), and has been approved by the authors' Institutional Review.

\section{References}

1. Verity R, Okell LC, Dorigatti I, Winskill P, Whittaker C, Imai N, et al. Estimates of the severity of coronavirus disease 2019: a modelbased analysis. Lancet Infect Dis 2020;20:669-77.

2. Martin Reeves PC-S, Whitaker K, Abraham M. Post-COVID era. Boston, MA: BCG Henderson Institute; 2020.

3. Nimmo B. Business continuity in a COVID-19 world. London, UK: KPMG International Limited (KPMG International); 2020.

4. Organization. WH. Corona virus disease 2019 (COVID-19): situation report-1; 2020. 20200121-sitrep-1-2019-ncov.pdf (who. int).

5. Organization World Health. Corona virus disease 2019 (COVID19): situation report-11. Geneva, Switzerland: World Health Organization; 2020. https://www.who.int/emergencies/ diseases/novel-coronavirus-2019/situation-reports.

6. Aminian A, Safari S, Razeghian-Jahromi A, Ghorbani M, Delaney CP. COVID-19 outbreak and surgical practice: unexpected fatality in perioperative period. Ann Surg 2020;272:e27-9.

7. Chopra V, Toner E, Waldhorn R, Washer L. How should US hospitals prepare for coronavirus disease 2019 (COVID-19)? Ann Intern Med 2020;172:621-3.

8. Desai A, Sachdeva S, Parekh T, Desai R. COVID-19 and cancer: lessons from a pooled meta-analysis. JCO Global Oncol 2020;6: 557-9.

9. Fong MW, Gao H, Wong JY, Xiao J, Shiu EY, Ryu S, et al. Nonpharmaceutical measures for pandemic influenza in nonhealthcare settings-social distancing measures. Emerg Infect Dis 2020;26:976-84.

10. Heymann DL, Shindo N. COVID-19: what is next for public health? Lancet 2020;395:542-5.

11. Leung K, Wu JT, Liu D, Leung GM. First-wave COVID-19 transmissibility and severity in China outside Hubei after control measures, and second-wave scenario planning: a modelling impact assessment. Lancet 2020;395:1382-93.

12. Lewnard JA, Lo NC. Scientific and ethical basis for social-distancing interventions against COVID-19. Lancet Infect Dis 2020;20:631-3.

13. Matrajt L, Leung T. Evaluating the effectiveness of social distancing interventions against COVID-19. Emerg Infect Dis 2020;26:1740-8.

14. McCall B. COVID-19 and artificial intelligence: protecting healthcare workers and curbing the spread. Lancet Digit Health 2020;2: e166-7.

15. Munster VJ, Koopmans M, van Doremalen N, van Riel D, de Wit E. A novel coronavirus emerging in China - key questions for impact assessment. N Engl J Med 2020;382:692-4.

16. Surgeons ACo. COVID-19 and surgery. Resources for the surgical community.

17. Burki TK. Cancer care in the time of COVID-19. Lancet Oncol 2020; 8:644-6.

18. Glehen O, Kepenekian V, Bouché O, Gladieff L, Honore C. Treatment of primary and metastatic peritoneal tumors in the Covid-19 pandemic. J Visc Surg 2020;157:S25-31. 
19. Lambertini M, Toss A, Passaro A, Criscitiello C, Cremolini C, Cardone $\mathrm{C}$, et al. Cancer care during the spread of coronavirus disease 2019 (COVID-19) in Italy: young oncologists' perspective. London, UK: BMJ Publishing Group Limited; 2020.

20. Liang W, Guan W, Chen R, Wang W, Li J, Xu K, et al. Cancer patients in SARS-CoV-2 infection: a nationwide analysis in China. Lancet Oncol 2020;21:335-7.

21. ESSO (European Society of Surgical Oncology). Statement on COVID-19. Advice for surgical oncologists on cancer service provision 2020. https://www.essoweb.org/news/essostatement-covid-19.

22. Gerstenhaber F, Grossman J, Lubezky N, Itzkowitz E, Nachmany I, Sever R, et al. Pancreaticoduodenectomy in elderly adults: is it justified in terms of mortality, long-term morbidity, and quality of life?. J Am Geriatr Soc 2013;61:1351-7.

23. Ministero de la Salute IMoH. Available from: http://www.salute.gov.it/ portale/nuovocoronavirus/dettaglioContenutiNuovoCoronavirus.jsp? lingua $=$ english \&id=5367\&area=nuovoCoronavirus \&menu=vuoto.

24. Health SMo. Daily report from Spanish ministry of health. Available from: https://covid19.isciii.es/.

25. Control CfD. Cases in U.S. Center for Disease Control [Accessed 19 April 2020].

26. Partners GHE. Daily COVID-19 global case summary report. GE Healthcare Command Centers; 2020.

27. COVID-19 projections. Institute for Healthcare Metrics and Evaluation; 2020.

28. Gallo G, Sturiale A, De Simone V, Mayol J. Epistemic networks on twitter: a new way to learn. J Invest Surg 2019;1-9. https://doi. org/10.1080/08941939.2019.1656787.

29. Mayol J, Otero J. Breaking international barriers: \#ColorectalSurgery is \#GlobalSurgery. Clin Colon Rectal Surg 2017;30:277-80.

30. Emanuel EJ, Persad G, Upshur R, Thome B, Parker M, Glickman A, et al. Fair allocation of scarce medical resources in the time of Covid-19. N Engl J Med 2020;382:2049-55.

31. Persad G, Wertheimer A, Emanuel EJ. Principles for allocation of scarce medical interventions. Lancet 2009;373:423-31.
32. Biddison LD, Berkowitz KA, Courtney B, De Jong CM, Devereaux $\mathrm{AV}$, Kissoon N, et al. Ethical considerations: care of the critically ill and injured during pandemics and disasters: CHEST consensus statement. Chest 2014;146:e145S-55S.

33. Surgeons ACo. ACS guidelines for triage and management of elective cancer surgery cases during the acute and recovery phases of coronavirus disease 2019 (COVID-19) pandemic; 2020.

34. Surgeons SSo. Documentos de posicionamiento y recomendaciones de la AEC en relación con la cirugía y COVID-19 2020. Available from: https://www.aecirujanos.es/Documentosde-posicionamiento-y-recomendaciones-de-la-AEC-en-relacioncon-la-cirugia-y-COVID19_es_1_152.html.

35. (SAGES) SoAGaES. Sages recommendations regarding surgical management $\mathrm{OF}$ colorectal cancer patients during the response to the COVID-19 crisis 2020. Available from: https://www.sages. org/recommendations-surgical-management-colorectal-cancercovid-19/.

36. Coloproctology ESo. ESCP endorses new recommendations regarding surgical response to COVID-19 crisis 2020. Available from: https://www.escp.eu.com/news/2088-escp-endorsesnew-recommendations-regarding-surgical-response-to-covid19-crisis.

37. Turaga K, Girotra S. Are we harming cancer patients by delaying their cancer surgery during the COVID-19 pandemic? Ann Surg 2020. https://doi.org/10.1097/SLA.0000000000003967 [Publish ahead of print].

38. Sud A, Jones ME, Broggio J, Loveday C, Torr B, Garrett A, et al. Collateral damage: the impact on cancer outcomes of the COVID19 pandemic. medRxiv 2020; Preprint.

39. Scheunemann LP, White DB. The ethics and reality of rationing in medicine. Chest 2011;140:1625-32.

40. Megginson LC. Lessons from Europe for American business. SW Soc Sci Q 1963;44:3-13.

Supplementary Material: The online version of this article offers supplementary material (https://doi.org/10.1515/pp-2020-0142). 